\title{
La cuestión territorial indígena en América Latina: algunas perspectivas desde Chile y Argentina
}

\author{
Nelson Martínez Berríos ${ }^{1}$, Bastien Sepúlveda ${ }^{2}$ \\ y Marcela Palomino-Schalscha ${ }^{3}$
}

La cuestión territorial indígena constituye sin lugar a dudas un tema de actualidad que, desde ya varias décadas, se vincula a las fuertes movilizaciones que han protagonizado diversas organizaciones y comunidades indígenas en distintas partes del planeta. En América Latina, más específicamente, este proceso se ha manifestado con particular énfasis desde el comienzo de los años 1990, en las perspectivas abiertas por las contracelebraciones del quinto centenario del "Descubrimiento". En efecto, en la mayoría de los países latinoamericanos, las reivindicaciones de numerosos pueblos y comunidades indígenas han venido cuestionando la realidad de una historia marcada por el sello del colonialismo y la usurpación. El "levantamiento indígena" liderado por la Confederación de Nacionalidades Indígenas del Ecuador (CONAIE) en 1990, las acciones del Ejército Zapatista de Liberación Nacional (EZLN) en Chiapas en 1994, o las movilizaciones pewenche contra el desarrollo hidroeléctrico en el Alto Biobío en Chile, son algunos ejemplos concretos de la vitalidad del movimiento indígena en la región.

Atendiendo esta situación, ya en 1971, las Naciones Unidas encomendaron al sociólogo

\footnotetext{
1 Escuela de Ciencias Ambientales, Universidad Católica de Temuco (Chile).E-mail: nmartinez@uct.cl

2 Centro Interdisciplinario de Estudios Interculturales e Indígenas-ICIIS, Pontificia Universidad Católica de Chile (Chile).

E-mail: bastien_sepulveda@yahoo.fr

3 School of Geography, Environment and Earth Sciences, Victoria University of Wellington (New Zealand).

E-mail: marcela.palomino-schalscha@vuw.ac.nz
}

ecuatoriano José Martínez Cobo la realización de un informe sobre el problema de la discriminación ejercida en contra de los pueblos indígenas. En dicho informe, publicado en 1986, J. Martínez Cobo subrayó la determinación de las poblaciones indígenas "de preservar, desarrollar y transmitir a futuras generaciones sus territorios ancestrales y su identidad étnica como base de su existencia continuada como pueblo, de acuerdo con sus propios patrones culturales, sus instituciones sociales y sus sistemas legales ${ }^{4}$. A partir de la publicación de ese estudio y de las sucesivas instancias de discusión en los organismos del sistema internacional de derechos humanos, las demandas por reconocimiento y protección de los territorios indígenas se han convertido en un importante foco de atención, particularmente en la literatura jurídica que pone de relieve el carácter fundamental de la relación que los pueblos indígenas mantienen con sus tierras ancestrales.

Esta relación, como lo sostienen los propios indígenas, estaría en la base de su existencia como pueblos, tal como lo establece un texto de la Comisión Interamericana de Derechos Humanos $(\mathrm{CIDH})$ de la Organización de Estados Americanos (OEA): "la protección del derecho a la propiedad de los pueblos indígenas sobre sus territorios ances-

\footnotetext{
4 MARTínez COBO, J. Estudio del problema de la discriminación contra las poblaciones indígenas - Vol. 5: Conclusiones, propuestas y recomendaciones. New York: Naciones Unidas, 1986 (ECN.4/ Sub.2/1986/7/ Add.4).
} 
trales es un asunto de especial importancia, porque su goce efectivo implica no solo la protección de una unidad económica sino la protección de los derechos humanos de una colectividad que basa su desarrollo económico, social y cultural en la relación con la tierra" ${ }^{\prime \prime}$.

Coincidentemente, el interés geográfico por la cuestión territorial indígena ha ido creciendo desde mediados de los años 1980 en distintas partes del mundo, manifestándose a través de una producción cada vez más importante, tanto en proyectos de investigación como en publicaciones. A modo de ilustración, desde 1984 a la fecha, una veintena de números temáticos dedicados a la cuestión indígena fueron publicados en distintas revistas especializadas del campo disciplinario, principalmente anglosajonas. Así también, grupos de trabajo especiales se han formado en la Association of American Geographers (AAG), la Canadian Association of Geographers (CAG) y en el Institute of Australian Geographers (IAG). En 2006, una comisión sobre los Derechos y Saberes de los Pueblos Indígenas ha sido también creada en la International Geographical Union (IGU).

Paradójicamente, la comunidad geográfica latinoamericana ha quedado globalmente al margen de esta dinámica. Debido probablemente a divisiones y tradiciones disciplinarias fuertemente marcadas, la cuestión indígena ha permanecido durante décadas en la academia latinoamericana como "dominio antropológico" por excelencia. Existe, sin embargo, en varios países de la región un interés incipiente desde la disciplina por abordar temáticas que, directa o indirectamente, se vinculan a cuestiones indígenas. Así lo demuestra, en particular, la publicación en 2012 de un primer dossier temático sobre "El territorio mapuche en Chile: perspectivas geográficas", en la Revista Geográfica del Sur (Vol. 3, Nº 1). Del mismo modo, en el transcurso de estos últimos años, se han ido abriendo espacios de debate en torno a la realidad terri-

\footnotetext{
$5 \mathrm{CIDH} / \mathrm{OEA}$. Derechos de los pueblos indígenas y tribales sobre sus tierras ancestrales y recursos naturales: normas y jurisprudencia del sistema interamericano de derechos humanos (OEA/Ser.L/V/II. Doc. 56/09, 30 diciembre 2009, Original: Español).
}

torial indígena latinoamericana, permitiendo a las "geografías indígenas" posicionarse en foros científicos regionales como el I Congreso Internacional de Pueblos Indígenas de América Latina (CIPIAL, Oaxaca, 2013) y el XV Encuentro de Geógrafos de América Latina (EGAL, La Habana, 2015).

En esta perspectiva también, se organizó el simposio "Geografías indígenas en Chile y América Latina", en el marco del XXXV Congreso Nacional y XX Internacional de la Sociedad Chilena de Ciencias Geográficas (SOCHIGEO), celebrado en Coyhaique del 4 al 8 de noviembre de 2014. Parte de las ponencias presentadas en ese simposio fueron reunidas en este dossier temático, con el cual Revista de Geografía Norte Grande no solo contribuye al desarrollo, en y desde América Latina, de un campo disciplinario ya instalado en otras regiones del mundo, sino que también permite posicionar la disciplina y precisar sus aportes con respecto a debates que animan al conjunto de las ciencias sociales desde ya varias décadas. A través de los artículos que lo componen, el presente número semitemático tiene por objetivo develar, reconocer y explicar la heterogeneidad de territorialidades y usos del espacio en contextos indígenas, analizando y comparando para tal efecto las acciones de "recuperación territorial" -y sus significados- con otras formas -rivales- de apropiación territorial, como son por ejemplo las actividades vinculadas al extractivismo.

Mientras nuevos focos de conflicto se siguen abriendo frente al incesante despliegue del neoliberalismo y a la multiplicación de proyectos extractivistas en muchas regiones de América Latina, resulta imperativo contar con una renovada experticia para abordar críticamente la cuestión territorial indígena. Experticia que, en pos de una reorientación de la acción pública en territorios indígenas, permita un mejor entendimiento tanto de las apuestas planteadas por el reconocimiento del derecho indígena al territorio, como de las recomposiciones territoriales contemporáneas y de las dinámicas que las sustentan. Abriéndose a distintas experiencias y situaciones territoriales relativas a pueblos indígenas en distintas regiones de Argentina y Chile, el presente volumen busca: 1) aportar a una mejor comprensión de las dinámicas 
territoriales contemporáneas, 2) avanzar en la comprensión y definición de la noción misma de "territorio indígena", y 3) aportar a un creciente cuerpo de literatura sobre geografías indígenas en América Latina.

Los siete artículos incluidos en este semitemático permiten destacar el interés que reviste el análisis geográfico y su marco de interpretación al momento de abordar la cuestión indígena. La perspectiva comparativa propuesta se fundamenta en la confrontación de estudios de caso que, basados en trabajos empíricos desde distintas regiones de Chile y Argentina, ahondan en la comprensión de las territorialidades indígenas contemporáneas. Desde el Altiplano chileno hasta la Patagonia argentina, pasando por el desierto de Atacama y la Araucanía andina, los siete trabajos que a continuación se presentan enlazan una gran heterogeneidad de espacios (rurales, urbanos, transnacionales, etc.) que caracterizan lo diverso, pero también lo complejo de las realidades territoriales indígenas contemporáneas.

Las contribuciones de Lorena Cañuqueo y Raúl Molina Otárola se adentran en el campo de la conflictividad territorial para explicar la relación -siempre compleja y no exenta de contradicciones- entre las comunidades indígenas y el Estado. Lorena Cañuqueo problematiza la injerencia del Estado en la configuración territorial mapuche en Argentina. Analizando la -dificultosa- aplicación de la Ley Nacional de Relevamiento Territorial en comunidades de la provincia de Río Negro, la autora devela las apuestas en juego en la definición de categorías espaciales que, "por ley" (de)limitan los territorios de las comunidades, no solo en su forma y cabida, sino también y sobre todo en su concepción.

En tanto, Raúl Molina Otárola aborda, desde una perspectiva diacrónica, los procesos intraétnicos que fueron configurando la construcción territorial aimara en el mundo andino. A partir del estudio de los conflictos territoriales entre las comunidades de ChuIluncane y Villablanca, en el altiplano de Tarapacá, el autor se adentra en las profundas contradicciones por dominio de tierras -ocasionadas principalmente por la superposición de títulos- que se desencadenaron a partir de la implementación de las políticas liberales del Estado chileno para constituir la propiedad aimara. Como resultado de esas políticas, y con el fin de asegurar la convivencia intercomunitaria, la territorialidad aimara se fue construyendo en base a constantes ciclos de conflicto y negociación que el autor analiza detalladamente.

Luego, los artículos de Viviana HuiliñirCurío, Marcela Palomino-Schalscha y Nelson Martínez Berríos sugieren nuevas avenidas de exploración para entender más específicamente la territorialidad pewenche en el Alto Biobío, en Chile. En primer lugar, Viviana Huiliñir-Curío, examina las redes de senderos utilizadas por los habitantes de las comunidades de Cauñicú y El Barco, haciendo un recorrido por la vivencia del territorio pewenche desde la geografía cultural. Este trabajo permite avanzar en la comprensión de las formas mediante las cuales las comunidades pewenche aseguran hoy el control territorial, dan nuevos significados a antiguas prácticas, y mantienen -reinventándola- una identidad cultural propia, vinculada al territorio.

Por su parte, Marcela Palomino-Schalscha analiza el desarrollo de la actividad turística en comunidades pewenche del valle del Queuco. El texto muestra que la "turistificación" de las comunidades indígenas es un acontecimiento no exento de tensiones. Pues, si bien el turismo puede ser visto como una nueva amenaza, puede también convertirse en una modalidad original de ejercer y reforzar el control territorial de las comunidades. Y lo interesante es que en estas recomposiciones, no solo actúan los turistas, sino también una serie de aliados diversos, tanto públicos como privados, incluyendo algunas ONGs e instituciones internacionales cuyas acciones calan hondo en el devenir territorial indígena.

Finalmente, la contribución de Nelson Martínez Berríos explora, a partir del examen de distintas prácticas sociales, una pluralidad de formas a través de las cuales los habitantes de la comunidad pewenche de Quinquén "ancestralizan" su territorio. Desde una perspectiva de geografía cultural, el autor postula que la "ancestralización" es un proceso continuo, vinculado a la forma de vida de las familias que conforman la comunidad, mediante nexos que mantienen la continuidad 
espacio-temporal de las relaciones sociales. El trabajo permite aportar así a una mejor comprensión de las distintas formas en que se expresa la territorialidad pewenche contemporánea.

Cerrando el tema central, las contribuciones de Brígida Baeza y de Bastien Sepúlveda y Paulina Zúñiga, dan cuenta de los importantes procesos migratorios a través de los cuales los territorios indígenas se han abierto y extendido a espacios no tradicionales que han incorporado a su estructura: urbanos -como en el caso mapuche en Chile, analizado por Sepúlveda y Zúñiga- y transnacionales -como en el caso de la comunidad quechua boliviana asentada en Caleta Oliva, presentado por Baeza-. Ambos trabajos ponen de relieve la variedad de mecanismos a través de los cuales las comunidades de migrantes se logran insertar y territorializar en estos nuevos espacios. En suma, lo que develan estos trabajos es una configuración territorial que se sostiene hoy en la articulación de una multiplicidad de lugares o, dicho de otro modo, plurilocalizada.

Tal como es tradición en Revista de Geografía Norte Grande, el presente número no está totalmente dedicado al tema central, sino que también acoge artículos sobre otros tópicos intentando asegurar una pluralidad de problemáticas territoriales. En esta ocasión, se divulgan cuatro trabajos y una reseña bibliográfica ajenos al tema principal.

El artículo de Felipe Link, Felipe Valenzuela y Luis Fuentes pretende analizar las posibilidades investigativas y metodológicas de la relación entre diferenciación social y el espacio urbano de Santiago de Chile, a través del uso de tipologías socioespaciales y su pertinencia en el contexto de los estudios tradicionales de segregación. Los autores concluyen que el análisis de tipologías socioespaciales constituye una herramienta útil para dar cuenta del tipo de diversidad social que caracteriza a cada subunidad territorial de la ciudad, permitiendo por ejemplo, identificar aquellos espacios de mayor homogeneidad social.

Por su parte, los geógrafos chilenos Pablo Sarricolea Espinoza y Hugo Romero Aravena estudian a distintas escalas temporales y es- paciales, las temperaturas, irregularidad de las precipitaciones y sequías en el Altiplano del norte de Chile. Señalan que desde el punto de vista geográfico, el área presenta un interesante gradiente norte sur de las variables de precipitación y temperatura, lo cual Ileva a concluir que no cabe esperar un comportamiento homogéneo de los climas altiplánicos, que justifique la aplicación de modelos geográficamente generalizados, lo que invalidaría a su vez estrategias de manejo, mitigación o adaptación al cambio climático que no consideren debidamente las particularidades de los ecosistemas y lugares específicos.

Félix Pillet Capdepón realiza un análisis de la evolución del paisaje a partir de la literatura de viajes, como precedente turístico. El autor plantea que en los espacios del turismo español, tanto de costa como de interior (de naturaleza rural, urbano e industrial) parece conveniente reivindicar como destino turístico el desarrollo de un turismo territorial o comarcal, que contemple en su conjunto la riqueza microespacial, pudiendo ser considerada también como Patrimonio Territorial. Un patrimonio basado en las unidades comarcales o agrupaciones comarcales, por ser los territorios más populares, mezcla de elementos naturales y culturales, materiales e inmateriales, y de contenidos literarios, algunas veces, con el fin de motivar el deseo de viajar o de hacer turismo local.

Por último, la investigación de María del Pilar Monserrat Pérez Hernández busca caracterizar las capacidades del Corredor Industrial El Bajío (CIB) en México a fin de identificar si este posee cualidades de un ambiente innovador. La metodología empleada consistió en hacer la revisión de literatura disponible y realizar una serie de entrevistas semiestructuradas a actores clave que participan en actividades de innovación en la región de estudio. A partir de esta información, se realizó el análisis cualitativo de las características productivas, científicas, tecnológicas y de innovación. Concluyendo que no ha habido estrategias sinérgicas para que esa identidad cultural, que ya existe en la región, se vuelva un factor para la explotación del conocimiento generado.

La reseña bibliográfica presentada en esta ocasión, es del texto "Planificación territorial 
y desarrollo del suelo empresarial en España", cuya directora es Paz Benito del Pozo. La obra hace referencia a los procesos territoriales y las estrategias seguidas en diferentes regiones septentrionales de España (Galicia, Asturias, Cantabria, Castilla y León y Navarra) referentes al suelo industrial y tecnológico, englobados aquí bajo el término de "suelo empresarial".
De esta manera, el № 62 de Revista de Geografía Norte Grande no solo busca contribuir a la creciente literatura sobre geografías indígenas en América Latina, sino también impulsar la apertura hacia nuevos enfoques que, desde campos disciplinarios como la geografía cultural, política y también histórica, invitan a desafiar las nociones convencionales de territorialidad, temporalidad y subjetividades. 
